\title{
Advancing Targeted Radionuclide Therapy Through the National Cancer Institute's Small Business Innovation Research Pathway
}

\author{
Kaveh Zakeri ${ }^{1,2}$, Deepa Narayanan ${ }^{3}$, Greg Evans ${ }^{3}$, Pataje Prasanna ${ }^{1}$, Jeffrey C. Buchsbaum ${ }^{1}$, Bhadrasain Vikram ${ }^{1}$, \\ and Jacek Capala ${ }^{1}$ \\ ${ }^{1}$ Radiation Research Program, Division of Cancer Treatment and Diagnosis, National Cancer Institute, Rockville, Maryland; \\ ${ }^{2}$ Department of Radiation Medicine and Applied Sciences, University of California San Diego, La Jolla, California; and ${ }^{3}$ Small \\ Business Innovation Research Development Center, National Cancer Institute, Bethesda, Maryland
}

\begin{abstract}
The Small Business Innovation Research (SBIR) and Small Business Technology Transfer (STTR) programs of the National Cancer Institute $(\mathrm{NCl})$ are congressionally mandated set-aside programs that provide research funding to for-profit small businesses for the development of innovative technologies and treatments that serve the public good. These two programs have an annual budget of $\$ 159$ million (in 2017) and serve as the NCl's main engine of innovation for developing and commercializing cancer technologies. In collaboration with the NCl's Radiation Research Program, the $\mathrm{NCl}$ SBIR Development Center published in 2015-2017 three separate requests for proposals from small businesses for the development of systemic targeted radionuclide therapy (TRT) technologies to treat cancer. TRT combines a cytotoxic radioactive isotope with a molecularly targeted agent to produce an anticancer therapy capable of treating local or systemic disease. This article summarizes the $\mathrm{NCI}$ SBIR funding solicitations for the development of TRTs and the research proposals funded through them.
\end{abstract}

Key Words: targeted radionuclide therapy; small business innovation research; oncology; radionuclide therapy; radiopharmaceuticals; theranostics

J Nucl Med 2019; 60:41-49

DOI: 10.2967/jnumed.118.214684

\section{$\mathbf{T}$} argeted radionuclide therapy (TRT) involves the precise delivery of radioactive isotopes to specific cells or molecular targets. Therapeutic radiopharmaceuticals consist of radionuclides attached to targeting agents, such as antibodies, proteins, peptides, or small molecules (1). Radioactive isotopes used in TRTs emit $\alpha$-particles (helium ions) or $\beta$-particles (electrons) that have a relatively short range of penetration in tissues, which allows delivery of lethal radiation doses targeted to cancer cells while sparing the surrounding normal tissues. Conjugating radionuclides to therapeutic agents used in molecularly targeted therapies has the additional advantage

Received May 15, 2018; revision accepted Jun. 18, 2018.

For correspondence or reprints contact: Jacek Capala, Radiation Research Program, Division of Cancer Treatment and Diagnosis, National Cancer Institute, 9609 Medical Center Dr., Rockville, MD 20850.

E-mail: capalaj@mail.nih.gov

Published online Jul. 20, 2018.

COPYRIGHT (C 2019 by the Society of Nuclear Medicine and Molecular Imaging. of producing synergistic damage to cancer cells, which can potentially overcome resistance to the parent drug.

The first clinical application of TRT was the treatment of thyroid cancer with radioactive iodine in the 1940s (2), and the field of TRT has since expanded with clinically approved indications for nonHodgkin lymphoma, bone metastases, and neuroendocrine tumors including neuroblastoma $(1,3)$. Two radiopharmaceuticals involving the ${ }^{90} \mathrm{Y}$ - and ${ }^{131}$ I-labeled anti-CD20 antibodies ${ }^{131}$ I-tositumomab (Bexxar; GlaxoSmithKline) and ${ }^{90}$ Y-ibritumomab tiuxetan (Zevalin; Spectrum Pharmaceuticals, Inc.) were approved by the Food and Drug Administration (FDA) for the treatment of non-Hodgkin lymphoma (4-6). The approval was based on evidence of improved response as compared with nonradiolabeled anti-CD20 antibodies. Building on the prior success of ${ }^{89} \mathrm{Sr}$ - and ${ }^{153} \mathrm{Sm}$-based therapies, ${ }^{223} \mathrm{RaCl}_{2}$ (Xofigo; Bayer) was tested in clinical trials and shown to improve survival for men with bone metastases from castrationresistant prostate cancer and has since been approved by the FDA for this indication $(7,8)$. Recently, on the basis of results of the phase III NETTER-1 trial showing evidence of overall survival benefit, ${ }^{177} \mathrm{Lu}$-DOTATATE (Lutathera; Advanced Accelerator Applications) has also been approved for the treatment of patients with somatostatin receptor-positive gastroenteropancreatic neuroendocrine tumors (9). These developments reinvigorated interest in TRT and novel therapeutic radiopharmaceuticals.

Here we describe the collaborative efforts of the National Cancer Institute (NCI) Radiation Research Program and Small Business Innovation Research (SBIR) Development Center to advance the field of TRT in cancer.

\section{SBIR PROGRAM}

The purpose of the SBIR program is to provide research funding through both SBIR grants and SBIR contracts to U.S.-owned and -operated small businesses (defined as $<500$ employees) to develop and commercialize novel technologies to prevent, diagnose, and treat cancer. Unlike other federal agencies, National Institutes of Health research-and-development contracts are not always aimed at procuring goods or services that will directly be used by the federal government. Although both methods provide research-and-development funding to small businesses to develop and commercialize their technologies, there are some critical differences between them. SBIR/Small Business Technology Transfer (STTR) grants are always investigator-initiated and are most often submitted through the SBIR/STTR omnibus funding 
announcement. NCI SBIR contracts are targeted funding opportunities for small businesses that are focused on specific research areas considered high-priority areas for the NCI. The NCI SBIR funding initiative and the general framework for novel drug development through this pathway have been previously described (10). Research-and-development contract funding is used to address specific community needs or to stimulate research in emerging fields that have a strong potential for commercialization. The contract topic on TRT was developed by the NCI Radiation Research Program in collaboration with the NCI SBIR Development Center and was selected after review by an NCI technology advisory group. The breakthrough success of ${ }^{223} \mathrm{RaCl}_{2}$ (Xofigo) in prostate cancer, leading to its approval by the FDA in 2013, and the need for further development of novel TRTs in new disease settings sparked the creation of funding opportunities through the SBIR program.

Each SBIR phase I contract received a budget of up to $\$ 300,000$ for 9 mo to perform initial proof-of-concept or feasibility studies, and SBIR phase II contract projects received up to $\$ 2,000,000$ each over 2 y to perform further research and development. Applicants also had the opportunity to simultaneously apply for phase I and II funding up front, through a mechanism termed fasttrack funding (https://sbir.nih.gov/apply/application-types). Phase I contracts for TRTs were for proof-of-concept and preliminary studies of novel radioisotopes, including feasibility, radiation dosimetry, pharmacokinetic/pharmacodynamics, and preliminary efficacy studies in suitable animal models. Unless they were awarded fast-track funding, phase I small-business contractors were invited to reapply for follow-on phase II funding and had to go through peer review again to be selected for phase II funding. Phase II contracts involved further research and development necessary for commercialization in preparation for FDA approval. Specific phase II aims typically involved manufacturing and scale-up, completion of investigational-new-drug (IND)-enabling studies for application with the FDA, and comparison of the novel agent to standard technologies or treatments.

In addition to funding opportunities, the NCI SBIR and NCI Radiation Research Program have hosted workshops to discuss challenges and identify solutions for small businesses developing TRT technologies $(11,12)$. A workshop titled "Facilitating the Development of Molecularly Targeted Radionuclide Therapy" was hosted on November 10, 2016, in Rockville, MD, in collaboration with the NCI Radiation Research Program and brought together contracted TRT small businesses with experts from the FDA, the U.S. Department of Energy, and the NCI (13). One objective of the workshop was to facilitate collaboration with the FDA early in the development of therapeutic radiopharmaceuticals and to establish a clear pathway leading to regulatory approval necessary to bring them to the clinic. The Department of Energy's National Isotope Development Center (https://isotopes.gov), which supports the production and distribution of radioactive isotopes, provided expert assistance to NCI SBIR awardees at the workshop.

\section{NCI SBIR-FUNDED TRT CONTRACTS}

NCI SBIR-funded research contracts for the development of TRTs from 2015 to 2017 are summarized in Tables 1 and 2. The funding information for these research proposals is publicly available at https://projectreporter.nih.gov/reporter.cfm. The NCI SBIR mechanism funded a total of 16 TRT contracts. Of these,
3 were fast-track awards, and a total of 5 companies have received phase II funding as of January 2018; however, companies funded in 2016 have just been invited to apply for phase II funding, and those funded in 2017 are just beginning their phase I research. Here we discuss how these research proposals aim to develop new TRTs that might improve the outcome of cancer treatment.

\section{Glioblastoma}

Glioblastomas are among the most aggressive cancers and are sorely in need of new treatment strategies. Molecular Targeting Technologies, Inc., received phase I funding to develop radioiodinated saposin $\mathrm{C}$-dioleoylphosphatidylserine nanovesicles for the treatment of glioblastoma. Saposin C-dioleoylphosphatidylserine has been shown to cross the blood-brain barrier and bind extracellular phosphatidylserine. Because radiation therapy causes phosphatidylserine to be upregulated on tumor cells, saposin C-dioleoylphosphatidylserine has enhanced anticancer potential in combination with radiation. This treatment strategy is of interest to clinicians because these agents cross the blood-brain barrier and the chemistry is well understood. Unlike chemotherapy, saposin C-dioleoylphosphatidylserine has sensitized hypoxic cells that, many believe, are responsible for failure of chemotherapy in glioblastoma (14).

\section{Melanoma}

Metastatic melanomas have a poor prognosis, but the unique proteins expressed by melanoma cells offer an opportunity for selective tumor targeting to improve treatment outcomes. The overexpression of the melanocortin 1 receptor (MCR1) on melanoma cells is an attractive target for melanoma-targeted therapy (15) and is sought after by SBIR contract recipients. The advantage of targeting melanoma-specific molecules is that this approach does not rely on specific-tumor genotypes or variability of a patient's immune response.

Modulation Therapeutics, Inc., is developing a ${ }^{225}$ Ac-based therapy for the treatment of ocular and advanced cutaneous melanoma. The company has developed a targeting molecule that binds MCR1 expressed on melanoma cells. The company attached DOTA to a MCR1 ligand and chelated the compound to ${ }^{225} \mathrm{Ac}$ to create ${ }^{225}$ Ac-DOTA-MCR1 ligand. After demonstrating that the compound has no overt toxicity and is efficacious in animal models of uveal and cutaneous melanoma, the company was awarded phase II funding.

Viewpoint Molecular Targeting, LLC, received phase I and II funding to determine the feasibility of a novel ${ }^{203 / 212} \mathrm{~Pb}$-labeled therapy for metastatic melanoma. The investigators attached ${ }^{203 / 212} \mathrm{~Pb}$ to DOTA-VMT-MCR1 and DOTA-RMX-glucosamine conjugate, which target melanoma-specific molecules. The objectives of the phase I SBIR were to determine the feasibility of radiosynthesis of ${ }^{212} \mathrm{~Pb}$-DOTA-RMX-glucosamine conjugate and ${ }^{212} \mathrm{~Pb}$-DOTAVMT-MCR1, evaluate the feasibility of ${ }^{212} \mathrm{~Pb}$-DOTA-RMXglucosamine conjugate and ${ }^{212} \mathrm{~Pb}-\mathrm{DOTA}-\mathrm{VMT}-\mathrm{MCR} 1$ therapy in melanoma-tumor-bearing mice, and determine the feasibility of a dual-targeted (glucose transporter 1/MCR1) bioconjugate for radionuclide imaging and therapy for metastatic melanoma. These studies would lay the necessary groundwork before IND-enabling studies and initiation of clinical trials.

RadImmune, Inc., received phase I funding to develop a radioimmunotherapy for metastatic melanoma based on a novel IgG to melanin. RadImmune previously completed a phase I 


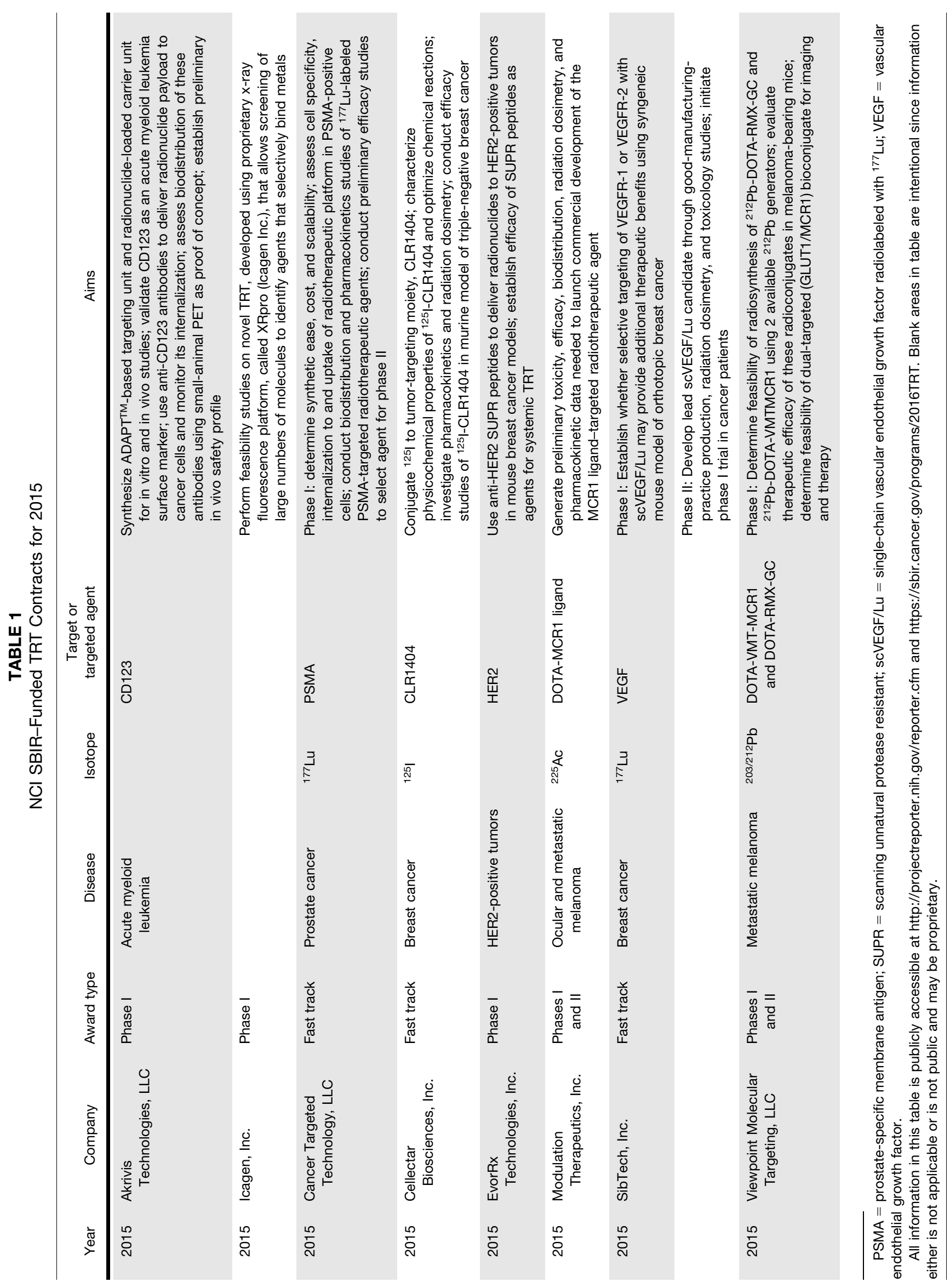




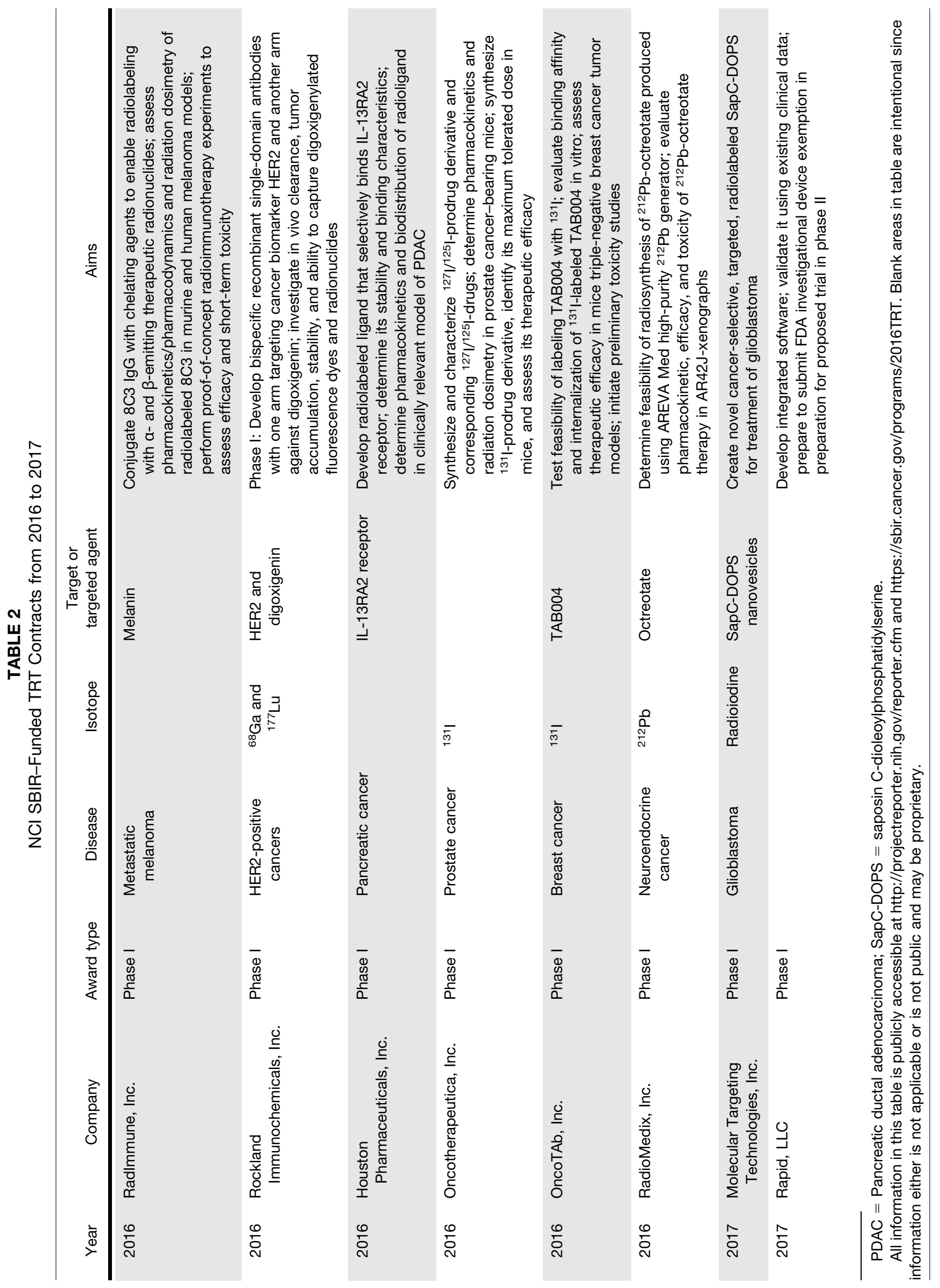

44 The Journal of Nuclear Medicine • Vol. 60 • No. 1 • January 2019 
clinical trial of an IgM antibody to melanin labeled with ${ }^{188} \mathrm{Re}$. However, the IgM isotype of the antibody was a barrier to clinical development. The aims of the phase I proposal were to conjugate $8 \mathrm{C} 3 \mathrm{IgG}$ with chelating agents to enable radiolabeling with $\alpha$ - and $\beta$-emitting radionuclides, perform pharmacokinetics/ pharmacodynamics studies and radiation dosimetry calculations in murine and human melanoma models, and perform proof-ofconcept experiments in murine and human melanoma models, including efficacy and short-term toxicity of the treatment. On the basis of these experiments, the most suitable radiolabeled form of IgG to melanin will be selected for future IND-enabling studies.

\section{Breast Cancer}

Triple-negative breast cancers do not express the targetable estrogen receptor or the human epidermal growth factor receptor 2 (HER2) that is expressed by other types of breast cancer. Consequently, triple-negative breast cancers have a worse prognosis, and therefore, it is necessary to identify novel targets. Cellectar Biosciences, Inc., obtained fast-track funding to develop CLR1404 (18-(p-iodophenyl)octadecyl phosphocholine) for patients with triple-negative breast cancer. CLR1404 is an alkyl phosphocholine that accumulates in lipid rafts, and since cancer cells have more lipid rafts than do normal cells, there is a selective mechanism for CLR1404 uptake (16). The aims of the proposal were to conjugate ${ }^{125}$ I to the tumor-targeting agent CLR1404 and define the physical and chemical properties of the compound to enable reliable drug synthesis; assess pharmacokinetics, radiation dosimetry, and normal-tissue toxicities in murine triple-negative breast cancer models; and conduct efficacy studies in mouse models.

OncoTAb, Inc., is also developing a radioimmunotherapy for triple-negative breast cancer. TAB004 is an antibody to the tumor form of the glycoprotein mucin 1 with high uptake in breast cancer tissues (17). The aims of the phase I proposal were to determine the feasibility of labeling the tumor-specific TAB004 antibody with ${ }^{131} \mathrm{I}$, evaluate the ability of the TRT to reduce tumors in mouse models of triple-negative breast cancer, and initiate preliminary toxicity studies.

SibTech, Inc., is collaborating with Johns Hopkins University to investigate a ${ }^{177} \mathrm{Lu}$ radiopharmaceutical for vascular endothelial growth factor receptor-mediated targeting of tumor vasculature. The company's data on mouse models of breast cancer demonstrate that the compound induces vascular regression, inhibits tumor growth, improves survival, and can be combined with chemotherapy. SibTech received fast-track funding to support the phase I goal of determining whether selective targeting of vascular endothelial growth factor receptor 1 or 2 is more advantageous in mouse models of orthotopic breast cancer. After the lead drug candidate is selected, the phase II aims are to achieve good-manufacturingpractice production, conduct dosimetry and toxicology studies, and undertake a phase I clinical trial.

\section{HER2-Positive Cancers}

Activating HER2 mutations have been identified in numerous solid cancers and represent an attractive target for TRT (18). For patients with HER2-positive breast and gastroesophageal cancers, HER2-directed therapy has become incorporated into standard-ofcare practice based on improved overall survival or disease-free survival (19-22). The benefit of HER2 therapy in other malignancies has yet to be firmly established, though active research is currently under way (18).

EvoRx Technologies, Inc., received phase I funding to use scanning unnatural-protease-resistant (SUPR) peptides as molecularly targeted agents for HER2-positive cancer. The advantage of SUPR peptides is that they bind targets with high specificity, leading to high tumor uptake, and the small size of SUPR peptides leads to high rates of clearance from the body as compared with antibodies and a consequent decrease in normal-tissue toxicity. The goals of the proposal were to use anti-HER2 SUPR peptides to deliver radionuclides to HER2-positive tumors in mouse breast cancer models and establish the efficacy of the anti-HER2 SUPR peptides.

Rockland Immunochemicals, Inc., is working in collaboration with Abzyme Therapeutics, LLC, and the University of Pittsburgh's Department of Radiology to develop a pretargeted radioimmunotherapy for HER2-positive cancers. Pretargeted radioimmunotherapy involves the administration of a cancer cell-targeting bispecific antibody followed, after the antibody binds to the tumor and clears from the blood, by a radiolabeled small-molecule ligand that binds to the antibody but is cleared rapidly from the rest of the body, reducing the exposure of normal tissues to radiation (23). The phase I aims are to develop bispecific recombinant single-domain antibodies with one arm targeting HER2 on cancer cells and the other arm targeting digoxigenin and to investigate in vivo clearance, tumor accumulation, stability, bioavailability, and the ability to capture digoxigenylated fluorescence dyes and radionuclides.

\section{Prostate Cancer}

Prostate cancer is one of the most common cancer diagnoses in men and a leading cause of cancer mortality. Traditional externalbeam radiotherapy and brachytherapy are effective for localized disease, but these modalities are less effective for patients with regional and distant disease. TRT has the potential to improve outcomes for these patients with disseminated prostate cancer. Oncotherapeutica, Inc., received phase I funding to develop a prodrug with ${ }^{131} \mathrm{I}$ attached for the treatment of prostate cancer. The prodrug is designed to be specifically activated within prostate cancer tumors. The specific aims were to synthesize the ${ }^{127} \mathrm{I}-$ prodrug/125I-prodrug derivative and corresponding 127IDS/125IDS, inject ${ }^{125} \mathrm{I}$-prodrug into mice with prostate cancer to determine tumor and normal-tissue uptake, and identify the maximum tolerated dose in mice and confirm therapeutic efficacy in mice with prostate cancer.

Cancer Targeted Technology, LLC, obtained fast-track funding to develop a ${ }^{177} \mathrm{Lu}$-based targeted radiotherapy for prostate cancer. The radionuclide is targeted to prostate-specific membrane antigen, which is a transmembrane protein expressed on prostate cells. The aims of phase I included identifying the lead ${ }^{177} \mathrm{Lu}$-labeled prostate-specific membrane antigen-targeting agent, conducting biodistribution and pharmacokinetic studies, and performing a preliminary assessment of efficacy. Phase II objectives include scale-up manufacturing and IND-enabling studies. Several of the phase II objectives will be conducted by subcontractors at the University of Pittsburgh.

\section{Pancreatic Cancer}

Houston Pharmaceuticals, Inc., received phase I funding to develop a radiolabeled ligand that binds the IL-13RA2 receptor in 


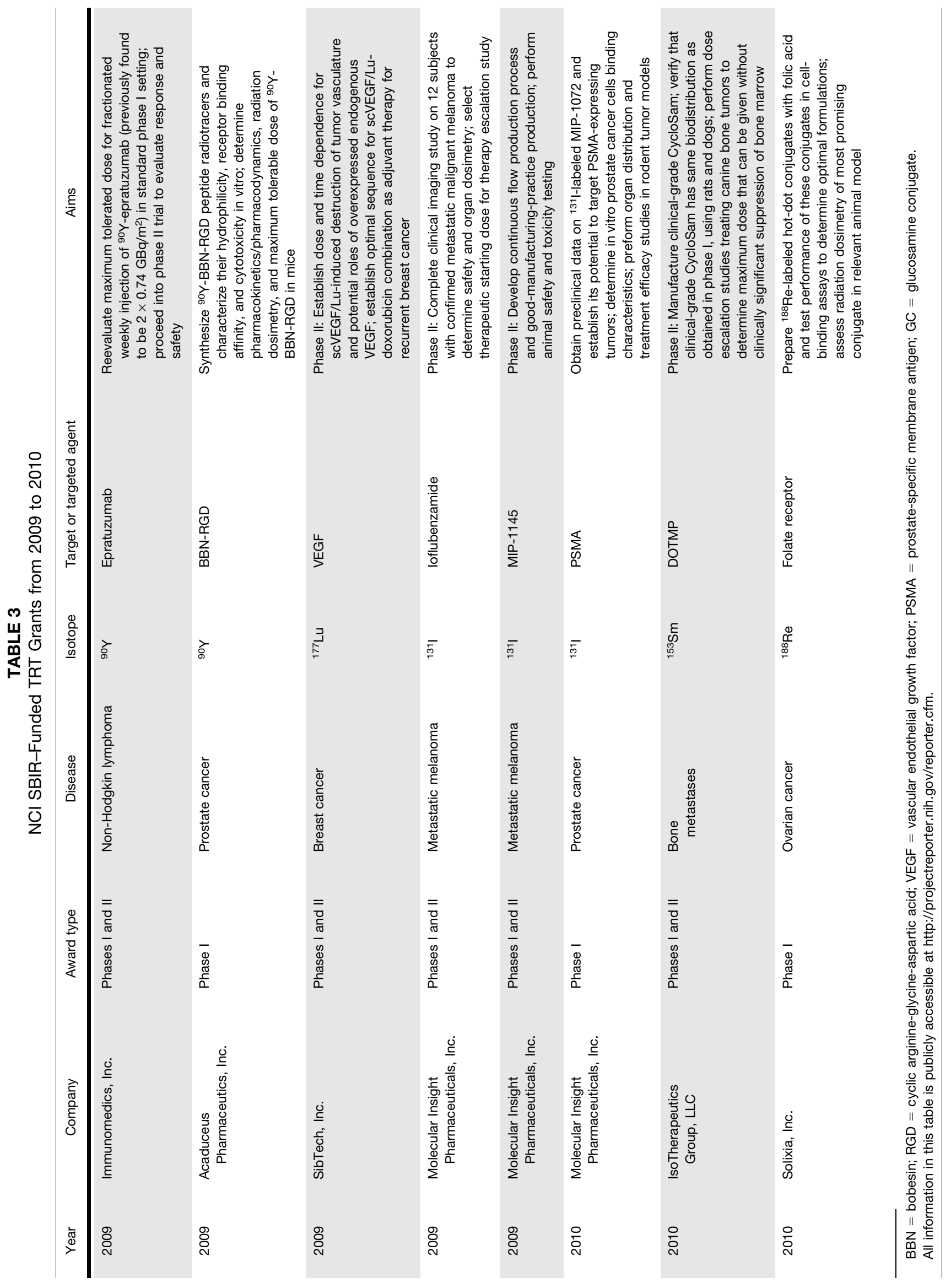

46 The Journal of Nuclear Medicine • Vol. 60 • No. 1 • January 2019 


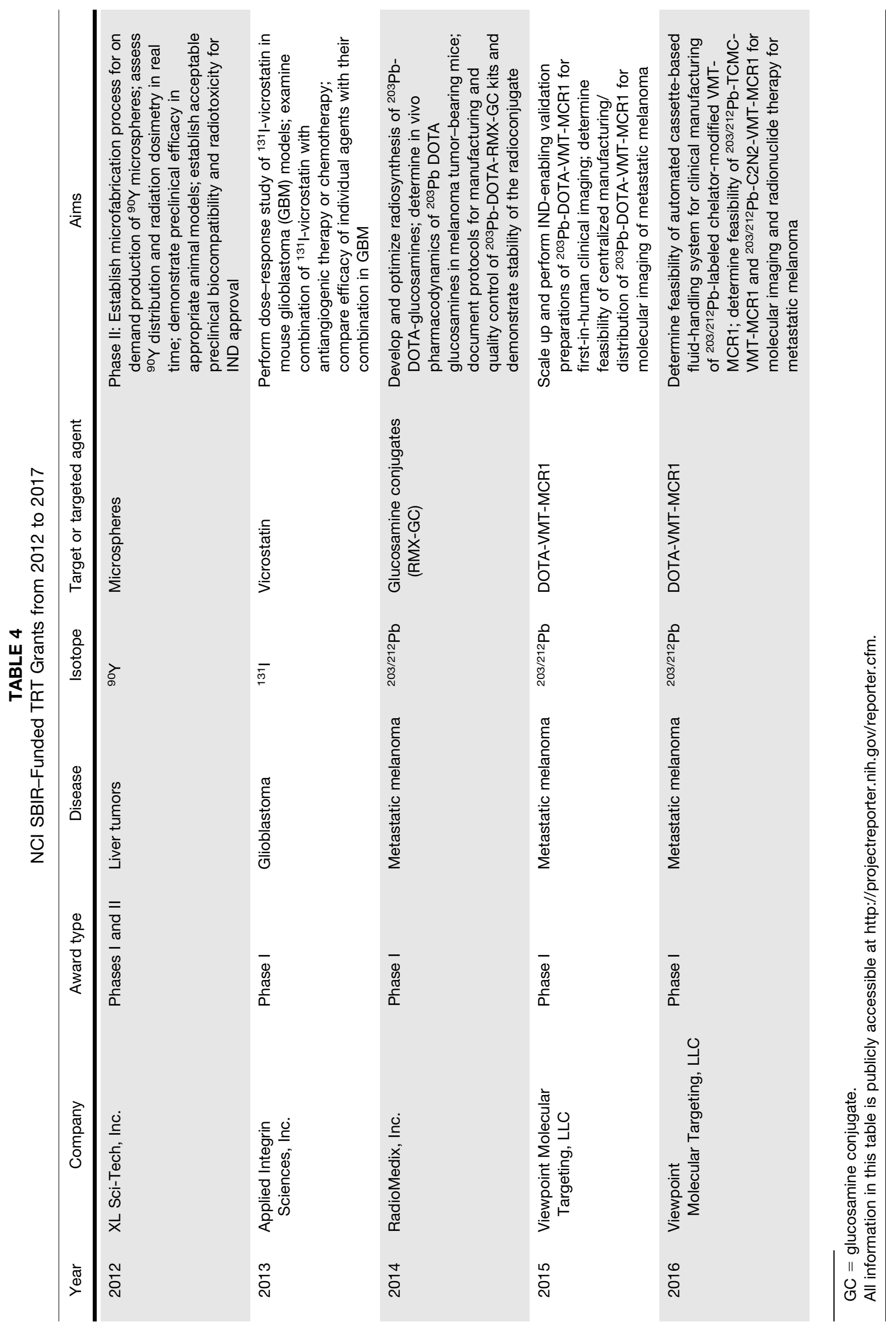


pancreatic cancer cells. IL-13RA2 is highly expressed by pancreatic cancer cells but not in normal tissue and thus is an attractive molecular target (24). The phase I objectives were to develop a radiolabeled ligand that selectively binds IL-13RA2 receptor; determine its binding affinity, specificity, and stability; and determine its pharmacokinetics and biodistribution in a model of pancreatic cancer.

\section{Neuroendocrine Tumors}

RadioMedix, Inc., received phase I funding to develop an $\alpha$-emitter-based TRT for neuroendocrine tumors. A $\beta$-emitterbased ${ }^{177} \mathrm{Lu}$-DOTATATE, which is currently used in the clinic, improves the response rate and progression-free survival compared with high-dose octreotide for patients with midgut neuroendocrine tumors (9). The investigators propose that an $\alpha$-emitter could overcome resistance developed to $\beta$-emitter radionuclide therapy. The goal of the phase I research proposal was to develop ${ }^{212} \mathrm{~Pb}$-octreotate. Specific aims included determining the feasibility of radiosynthesis and evaluating the pharmacokinetics, efficacy, and toxicity of ${ }^{212} \mathrm{~Pb}$-octreotate in xenographs.

\section{TRT Dosimetry}

Personalized dosimetry for TRT involves assessment of medical images, radionuclide pharmacokinetics, and patientspecific anatomy to determine the optimal therapy for an individual patient $(25,26)$. The lack of individualized dosimetry could be a barrier to the success of TRT, since different patients may have different pharmacokinetics and accumulation of radionuclides in tumor and normal tissues, leading to different biologic responses. Dosimetry measurements have the potential to affect the NCI's TRT program because the definition of dose can be refined and expanded to include multiple modalities and, ultimately, a biologic response that is individualized. Rapid, LLC, received phase I funding to develop dosimetry software for TRTs that can be used in combination with external-beam radiotherapy. The aims of the proposal were to create and validate the software using existing clinical data and to submit an investigational device exemption with the FDA in preparation for a phase II clinical trial.

\section{SBIR GRANTS}

Approximately $75 \%$ of the NCI SBIR Development Center award budget is used to support SBIR/STTR grant funding. SBIR grants are investigator-initiated solicitations typically received through the National Institutes of Health omnibus grant solicitation. The NCI has awarded several SBIR grants for the development of TRTs (Tables 3 and 4). The funding information for SBIR grants is also publicly available at https://projectreporter.nih.gov/ reporter.cfm. Viewpoint Molecular Targeting and RadioMedix have received both SBIR contracts and grants for the development of TRT, indicating the multiple avenues of funding available to companies.

\section{CONCLUSION}

Expanding the scope of TRT in cancer could yield meaningful improvements for patients. The NCI Radiation Research Program has partnered with the SBIR program to help overcome historical barriers to the translation of TRT from the bench to the clinic. It is interesting to note that just in the past $3 \mathrm{y}$, the NCI's SBIR Development Center has funded as many TRT projects and companies through SBIR contracts as through SBIR grants in the past $9 \mathrm{y}$-indicating that SBIR contracts were successful in stimulating small-business involvement in emerging areas. The solicitation of TRT contracts has increased interest in the field and facilitated stronger collaborations between academia and industry. The SBIR program has provided funding for promising TRTs in the preclinical stage of development so that necessary INDenabling studies can be completed. The NCI is encouraging companies to obtain feedback from the FDA early in the course of drug development to avoid potential pitfalls and to accelerate the path to commercialization. These collaborative efforts between government, industry, and academia maximize the opportunities to develop novel TRTs for patients with cancer.

\section{DISCLOSURE}

No potential conflict of interest relevant to this article was reported.

\section{REFERENCES}

1. Gill MR, Falzone N, Du Y, Vallis KA. Targeted radionuclide therapy in combined-modality regimens. Lancet Oncol. 2017;18:e414-e423.

2. Seidlin SM, Marinelli LD, Oshry E. Radioactive iodine therapy; effect on functioning metastases of adenocarcinoma of the thyroid. J Am Med Assoc. 1946;132: 838-847.

3. Zukotynski K, Jadvar H, Capala J, Fahey F. Targeted radionuclide therapy: practical applications and future prospects. Biomark Cancer. 2016;8(suppl 2): 35-38.

4. Shimoni A, Avivi I, Rowe JM, et al. A randomized study comparing yttrium-90 ibritumomab tiuxetan (Zevalin) and high-dose BEAM chemotherapy versus BEAM alone as the conditioning regimen before autologous stem cell transplantation in patients with aggressive lymphoma. Cancer. 2012;118: 4706-4714.

5. Morschhauser F, Radford J, Van Hoof A, et al. ${ }^{90}$ Yttrium-ibritumomab tiuxetan consolidation of first remission in advanced-stage follicular non-Hodgkin lymphoma: updated results after a median follow-up of 7.3 years from the international, randomized, phase III First-Line Indolent trial. J Clin Oncol. 2013;31:1977-1983.

6. Leahy MF, Seymour JF, Hicks RJ, Turner JH. Multicenter phase II clinical study of iodine-131-rituximab radioimmunotherapy in relapsed or refractory indolent non-Hodgkin's lymphoma. J Clin Oncol. 2006;24:4418-4425.

7. Parker C, Nilsson S, Heinrich D, et al. Alpha emitter radium-223 and survival in metastatic prostate cancer. $N$ Engl J Med. 2013;369:213-223.

8. Saad F, Carles J, Gillessen S, et al. Radium-223 and concomitant therapies in patients with metastatic castration-resistant prostate cancer: an international, early access, open-label, single-arm phase $3 \mathrm{~b}$ trial. Lancet Oncol. 2016;17:1306-1316.

9. Strosberg J, El-Haddad G, Wolin E, et al. Phase 3 trial of ${ }^{177}$ Lu-DOTATATE for midgut neuroendocrine tumors. N Engl J Med. 2017;376:125-135.

10. Prasanna PGS, Narayanan D, Hallett K, et al. Radioprotectors and radiomitigators for improving radiation therapy: the small business innovation research (SBIR) gateway for accelerating clinical translation. Radiat Res. 2015;184:235-248.

11. Fahey F, Zukotynski K, Capala J, Knight N; organizing committee, contributors, and participants of NCI/SNMMI Joint Workshop on Targeted Radionuclide Therapy. Targeted radionuclide therapy: proceedings of a joint workshop hosted by the National Cancer Institute and the Society of Nuclear Medicine and Molecular Imaging. J Nucl Med. 2014;55:337-348.

12. Fahey F, Zukotynski K, Jadvar H, Capala J; organizing committee, contributors, and participants of the second NCI-SNMMI workshop on targeted radionuclide therapy. Proceedings of the second NCI-SNMMI workshop on targeted radionuclide therapy. J Nucl Med. 2015;56:1119-1129. 
13. Canaria CA, Lisa Yeom M, Capala J, Narayanan D. Proceedings of the NCI SBIR workshop on molecularly targeted radionuclide therapy. J Nucl Med. 2018; 59(6): $13 \mathrm{~N}-14 \mathrm{~N}$.

14. Wojton J, Chu Z, Mathsyaraja H, et al. Systemic delivery of SapC-DOPS has antiangiogenic and antitumor effects against glioblastoma. Mol Ther. 2013;21: $1517-1525$.

15. Raposinho PD, Correia JDG, Oliveira MC, Santos I. Melanocortin-1 receptor-targeting with radiolabeled cyclic $\alpha$-melanocyte-stimulating hormone analogs for melanoma imaging. Biopolymers. 2010;94:820-829.

16. Morris ZS, Weichert JP, Saker J, et al. Therapeutic combination of radiolabeled CLR1404 with external beam radiation in head and neck cancer model systems. Radiother Oncol. 2015;116:504-509.

17. Roy LD, Dillon LM, Zhou R, et al. A tumor specific antibody to aid breast cancer screening in women with dense breast tissue. Genes Cancer. 2017;8:536-549.

18. Connell CM, Doherty GJ. Activating HER2 mutations as emerging targets in multiple solid cancers. ESMO Open. 2017;2:e000279.

19. Swain SM, Baselga J, Kim S-B, et al. Pertuzumab, trastuzumab, and docetaxel in HER2-positive metastatic breast cancer. N Engl J Med. 2015;372: 724-734.

20. Cameron D, Piccart-Gebhart MJ, Gelber RD, et al. 11 years' follow-up of trastuzumab after adjuvant chemotherapy in HER2-positive early breast cancer: final analysis of the HERceptin Adjuvant (HERA) trial. Lancet. 2017;389:1195-1205.

21. Slamon DJ, Leyland-Jones B, Shak S, et al. Use of chemotherapy plus a monoclonal antibody against HER2 for metastatic breast cancer that overexpresses HER2. N Engl J Med. 2001;344:783-792.

22. Bang Y-J, Van Cutsem E, Feyereislova A, et al. Trastuzumab in combination with chemotherapy versus chemotherapy alone for treatment of HER2positive advanced gastric or gastro-oesophageal junction cancer (ToGA): a phase 3, open-label, randomised controlled trial. Lancet. 2010;376: 687-697.

23. Meredith RF, Buchsbaum DJ. Pretargeted radioimmunotherapy. Int J Radiat Oncol Biol Phys. 2006;66(suppl):S57-S59.

24. Sai KKS, Sattiraju A, Almaguel FG, et al. Peptide-based PET imaging of the tumor restricted IL13RA2 biomarker. Oncotarget. 2017;8:5099751007.

25. Eberlein U, Cremonesi M, Lassmann M. Individualized dosimetry for theranostics: necessary, nice to have, or counterproductive? J Nucl Med. 2017;58(suppl 2): 97S-103S

26. Sgouros G, Kolbert KS, Sheikh A, et al. Patient-specific dosimetry for ${ }^{131} \mathrm{I}$ thyroid cancer therapy using ${ }^{124} \mathrm{I}$ PET and 3-dimensional-internal dosimetry (3D-ID) software. J Nucl Med. 2004;45:1366-1372. 